\title{
Potential proinflammatory effects of hydroxyapatite nanoparticles on endothelial cells in a monocyte-endothelial cell coculture model
}

This article was published in the following Dove Press journal:

International Journal of Nanomedicine

II March 2014

Number of times this article has been viewed

Xin Liu

Jiao Sun

Shanghai Biomaterials Research and Testing Center, Shanghai Key Laboratory of Stomatology, Ninth People's Hospital, Shanghai Jiao

Tong University School of Medicine, Shanghai, People's Republic of China

Correspondence: Jiao Sun No 427, Ju-men Road, Shanghai 200023 , People's Republic of China Tel +862163034903

$\mathrm{Fax}+862163011643$

Email jiaosun59@yahoo.com

\begin{abstract}
Currently, synthetic hydroxyapatite nanoparticles (HANPs) are used in nanomedicine fields. The delivery of nanomedicine to the bloodstream exposes the cardiovascular system to a potential threat. However, the possible adverse cardiovascular effects of HANPs remain unclear. Current observations using coculture models of endothelial cells and monocytes with HANPs to mimic the complex physiological functionality of the vascular system demonstrate that monocytes could play an important role in the mechanisms of endothelium dysfunction induced by the exposure to HANPs. Our transmission electron microscopy analysis revealed that both monocytes and endothelial cells could take up HANPs. Moreover, our findings demonstrated that at a subcytotoxic dose, HANPs alone did not cause direct endothelial cell injury, but they did induce an indirect activation of endothelial cells, resulting in increased interleukin- 6 production and elevated adhesion molecule expression after coculture with monocytes. The potential proinflammatory effect of HANPs is largely mediated by the release of soluble factors from the activated monocytes, leading to an inflammatory response of the endothelium, which is possibly dependent on p38/c-Jun N-terminal kinase, and nuclear factorkappa B signaling activation. The use of in vitro monocyte-endothelial cell coculture models for the biocompatibility assessment of HANPs could reveal their potential proinflammatory effects on endothelial cells, suggesting that exposure to HANPs possibly increases the risk of cardiovascular disease.
\end{abstract}

Keywords: coculture, monocytes, endothelial cells, inflammation, hydroxyapatite nanoparticles

\section{Introduction}

Synthetic hydroxyapatite (HA) $\left(\mathrm{Ca}_{10}\left[\mathrm{PO}_{4}\right]_{6}[\mathrm{OH}]_{2}\right)$, a typical bioceramic with good osteoconductive and osteoinductive capabilities, has been used clinically for many years. ${ }^{1}$ Currently, nanotechnology has entered the field of biomaterials, resulting in the rapid development of nano-based HA. Due to their better bioactivity, their excellent capacity to penetrate cell membranes, and their increased circulation time, HA nanoparticles (HANPs) have gradually garnered significant interest in various medical fields, such as bone tissue engineering, cardiovascular graft coating, contrast agent synthesis, drug delivery, and gene therapy. ${ }^{2-5}$ In these cases of therapeutic and diagnostic application, HANPs may become systemically available, which increases the risk of their exposure to the blood vessels. It has been proposed that atherosclerotic complications may occur with the continued use of HANPs., ${ }^{3,6}$ Thus, in terms of human health, the main potential adverse effect of HANPs on the cardiovascular system needs to be carefully assessed before reaching the clinical application stage. 
It is well known that synthetic nanoparticles (NPs) can enter the vascular system intentionally by injection in the form of nanomedicines or nanodiagnostics. ${ }^{7}$ Accordingly, endothelial cells (ECs), which form the inner cellular lining of the entire cardiovascular system, have direct contact with these NPs. In addition to ECs, NPs may simultaneously encounter circulating immune cells upon introduction into the blood circulation. Monocytes/macrophages are the body's first line of defense and are recognized as important contributors to atherosclerosis via interactions with ECs. Thus, considering the fundamental role exerted by both ECs and monocytes in cardiovascular events, to understand the interaction of monocytes and ECs, both with each other and with NPs, it is very important to assess NP-induced cardiovascular effects. In recent years, several toxicology studies have demonstrated that diesel particulates can indirectly activate ECs via exposure to macrophages, with even more profound effects than those generated by direct exposure to ECs. ${ }^{8-10}$ Our current studies also found that silica NPs could significantly augment proinflammatory and procoagulant responses in ECs through monocyte-EC interactions. ${ }^{11}$ However, to the best of our knowledge, most studies are still focused on the direct biological response of engineered HANPs to monocultures of cells in the endothelium or the immune system; ${ }^{5,12-18}$ as such, the coculture of monocytes and ECs with HANPs has received little attention in this regard.

In this study, to model an in vivo vascular microenvironment when HANPs enter into systemic circulation, we established an in vitro coculture model using THP-1 cells (monocytes) and human umbilical vein ECs (HUVECs). By utilizing this in vitro system, we investigated both the direct and the integrated/indirect effects of NPs on ECs in the presence or absence of THP-1 cells to evaluate the potential cardiovascular toxicity of HANPs. First, HANPs were synthesized, and their physiochemical characteristics were studied. Subsequently, HANP biocompatibility was assessed at the level of specific features, including cell viability, cellular uptake, cytokine production, and cell adhesion molecule (CAM) expression in HUVECs and THP-1 cells in monoculture and in HUVECs/THP-1 cells cocultured with HANPs. Moreover, the proinflammatory effects of HANPs on ECs were compared following their exposure to direct contact with the coculture, and after exposure to monocytederived soluble factors. Finally, to investigate the signaling pathway activated by HANPs in ECs cocultured with THP-1 cells, we measured the stimulation of mitogen-activated protein kinases (MAPKs) and nuclear factor-kappa B (NF$\kappa B)$ in HUVECs.

\section{Materials and methods Preparation and characterization of HANPs}

HANPs were synthesized using a chemical precipitation method according to our previously published procedures. ${ }^{19}$ Briefly, the $\mathrm{pH}$ of a $\mathrm{Ca}\left(\mathrm{NO}_{3}\right)_{2}$ solution was adjusted to 12 by adding a concentrated ammonia solution, and an $\left(\mathrm{NH}_{4}\right)_{2} \mathrm{HPO}_{4}$ solution $(\mathrm{pH}=12)$ was added dropwise during vigorous stirring. A voluminous precipitate was formed. The reaction mixture was gently boiled for 10 minutes. The precipitate was allowed to settle, and it was subsequently separated from the supernatant by centrifugation. The HA precipitate was washed with distilled water, frozen at $-20^{\circ} \mathrm{C}$, and lyophilized. The size and shape of the HANPs were examined using transmission electron microscopy (TEM) (JEOL, Tokyo, Japan). The structure of HANPs was examined by X-ray diffraction (XRD) using a RINT2000 vertical goniometer (Rigaku Corporation, Tokyo, Japan). The powder was examined with $\mathrm{Ni}$ filtered $\mathrm{CuK} \alpha$ radiation generated at $40 \mathrm{kV}$ and $100 \mathrm{~mA}$. The powders were scanned from $10^{\circ}-80^{\circ} 2 \theta$ with a scan speed of $2^{\circ}$ per minute. The peak of the obtained XRD pattern was compared with standard HA in the Joint Committee on Power Diffraction Standards file available in software (No 09-0432). Energy-dispersive X-ray spectroscopy can be used to find the chemical composition of materials. Thus, for the elemental analysis, a Hitachi S-2600N-type scanning electron microscope (Hitachi Ltd, Tokyo, Japan) was equipped with an energy dispersive X-ray attachment (EDAX Inc., Mahwah, NJ, USA) to create element composition spectra. The hydrodynamic size and surface charge (zeta potential) of the HANP dispersions were characterized using the Zetasizer Nano ZS (Malvern Instruments, Malvern, UK) utilizing dynamic light scattering and electrophoretic light scattering, respectively. The specific surface area of the HANPs was determined by the BrunauerEmmett-Teller method using a surface area analyzer (ASAP 2020; Micromeritics Instrument Corporation, Norcross, GA, USA) after pre-preparation of samples by heating at $200^{\circ} \mathrm{C}$ in a stream of $\mathrm{N}_{2}$ in excess of 24 hours.

\section{Endotoxin measurement}

The endotoxin content in the HANP dispersions was tested using the Limulus amebocyte lysate kinetic chromogenic assay (Sigma-Aldrich, St Louis, MO, USA). The endotoxin content in the HANP dispersions used in the study was below $1 \mathrm{EU} / \mathrm{mL}$.

\section{Cell preparation and culture}

HUVECs were isolated from freshly obtained human umbilical cord using our previously published method. ${ }^{20}$ Briefly, the 
umbilical vein was rinsed three times with phosphate buffered saline (PBS) containing $100 \mu \mathrm{g} / \mathrm{mL}$ of penicillin/streptomycin $\left(\right.$ Gibco $^{\circledR}$; Life Technologies, Carlsbad, CA, USA), filled with $0.1 \%$ collagenase I (Sigma-Aldrich) and incubated for 15 minutes at $37^{\circ} \mathrm{C}$. After harvesting, the ECs were placed in $75 \mathrm{~cm}^{2}$ tissue culture flasks (Corning Incorporated, Corning, NY, USA) and grown in EC medium (ScienCell Research Labs, Carlsbad, CA, USA). HUVECs between the third and sixth passages were used in our experiments. The phenotype of the ECs was confirmed by performing immunofluorescence using monoclonal antibodies for the von Willebrand factor (vWF) (ShangHai ChangDao Biotech Co, Ltd, Shanghai, People's Republic of China).

Human monocytes (THP-1 cells) were purchased from the Cell Bank of the Chinese Academy of Sciences (Shanghai, People's Republic of China) and were cultured in Roswell Park Memorial Institute (RPMI) 1640 medium (Gibco ${ }^{\circledR}$; Life Technologies) supplemented with $10 \%$ fetal bovine serum (Biochrom AG, Berlin, Germany) and $100 \mu \mathrm{g} / \mathrm{mL}$ of penicillin/streptomycin.

For the coculture of monocytes and HUVECs, THP-1 cells $\left(1 \times 10^{6}\right.$ cells $\left./ \mathrm{mL}\right)$ were added onto confluent HUVEC layers $\left(5 \times 10^{5}\right.$ cells $\left./ \mathrm{mL}\right)$ in six-well plates. Contact cocultures were performed in the presence or absence of HANPs for 24 hours. Meanwhile, to prevent direct cell contact between monocytes and HUVECs, at the end of the 24-hour incubation, the supernatants were collected, cleared of cells by centrifugation, and transferred to stimulate the HUVECs or monocytes for 24 hours and cultured in parallel.

\section{Cell viability assays}

Cell viability was measured by assessment of mitochondrial function using the CellTiter $96^{\circledR}$ AQueous One Solution assay (Promega Corporation, Madison, WI, USA). The solution reagent contains a tetrazolium compound (3-[4, 5-dimethylthiazol-2-yl]-5-[3-carboxymethoxyphenyl]-2[4-sulfophenyl]-2H-tetrazolium, inner salt; MTS) and an electron coupling reagent (phenazine ethosulfate). After seeding the cells for 24 hours, differing concentrations of HANPs were added to the 96-well plates. The cultures were further incubated for 24 hours, and $20 \mu \mathrm{L}$ of MTS agent was then directly added to each well. After a 4-hour incubation period, the absorbance of formazan was measured at $490 \mathrm{~nm}$ using a microplate reader (Wellscan MK3, Labsystems Dragon, Inc., Helsinki, Finland).

\section{Particle uptake}

To determine the cellular uptake and localization of the particles, HUVECs and THP-1 cells were individually exposed to HANPs $(100 \mu \mathrm{g} / \mathrm{mL})$ for 24 hours and analyzed by TEM. After incubation for 24 hours with HANPs, the cells were washed with a PBS solution, trypsinized, and centrifuged. Next, the cell pellets were fixed in a $0.1 \mathrm{M}$ PBS solution containing $2.5 \%$ glutaraldehyde for 4 hours. The cells were dehydrated through a series of ethanol washes $(70 \%$ for 15 minutes, $90 \%$ for 15 minutes, and $100 \%$ for 15 minutes, twice) and embedded in Epon-Araldite resin (polymerization at $65^{\circ} \mathrm{C}$ for 15 hours). Thin sections containing the cells were placed on the grids and stained for 1 minute each with $4 \%$ uranyl acetate (in acetone: water, 1:1) and 0.2\% Reynolds' lead citrate (in water), air-dried, and imaged under TEM.

\section{Cytokine measurement}

For cytokine analysis (interleukin [IL]-6, IL-8, IL-1 $\beta$, and tumor necrosis factor-alpha [TNF- $\alpha]$ ), the supernatants of HUVECs or THP-1 cells in cocultures or monocultures exposed to HANPs $(100 \mu \mathrm{g} / \mathrm{mL})$ were collected after 24 hours, immediately centrifuged to remove the cells, and then frozen at $-80^{\circ} \mathrm{C}$ until the analysis was performed. The amounts of IL-6, IL-8, IL-1 $\beta$, and TNF- $\alpha$ were quantified with an immunoassay kit (R\&D Systems, Inc., Minneapolis, MN, USA) according to the manufacturer's instructions.

\section{Immunofluorescence flow cytometry}

The levels of surface markers expressed on HUVECs were assessed using flow cytometry. After 24 hours of coculture or monoculture in the absence or presence of HANPs $(100 \mu \mathrm{g} / \mathrm{mL})$, HUVECs were released from the wells after washing with PBS. The following mouse antihuman monoclonal antibodies were used: intercellular adhesion molecule (ICAM-1) (cluster of differentiation [CD]54-PE; eBioscience, Inc., San Diego, CA, USA); vascular CAM-1 (VCAM-1) (CD106 fluorescein isothiocyanate isomer 1; BD Biosciences, San Jose, CA, USA); E-selectin (CD62E-APC; BD Biosciences); and vWF (BD Biosciences). Subsequently, HUVECs were collected and labeled with the abovementioned specific antibodies at room temperature for 45 minutes in the dark, washed extensively, and then subsequently fixed with $1 \%$ paraformaldehyde. All samples were analyzed using a FACScan ${ }^{\mathrm{TM}}$ flow cytometer (BD, Franklin Lakes, NJ, USA). The data were analyzed with CellQuest ${ }^{\mathrm{TM}}$ software (BD Biosciences).

\section{Western blot analysis}

Protein expression was determined using Western blot. Briefly, following the incubation periods, HUVECs were washed once with ice-cold PBS and lysed in ice-cold radioimmunoprecipitation assay buffer (Applygen Technologies Inc., Beijing, People's Republic of China) containing $1 \mathrm{mM}$ of 
phenylmethylsulfonyl fluoride (Sigma-Aldrich) and a phosphatase inhibitor cocktail (Sigma-Aldrich) for 30 minutes. After centrifuging the lysates at $12,000 \mathrm{rpm}$ at $4^{\circ} \mathrm{C}$ for 10 minutes, the supernatants were collected and stored at $-80^{\circ} \mathrm{C}$ for future use. The protein concentrations of these extracts were determined by performing a bicinchoninic acid protein assay (Thermo Fisher Scientific, Waltham, MA, USA). Equal amounts of protein samples $(40 \mu \mathrm{g})$ were separated using 10\% sodium dodecyl sulfate-polyacrylamide gels (SDS-PAGE) and subsequently transferred onto nitrocellulose membranes (Amersham plc, Amersham, UK). Membranes were blocked with 5\% nonfat milk buffer and incubated with anti-p-p38, anti-p-p-c-Jun N-terminal kinase (JNK), anti-JNK (1:1,000, rabbit polyclonal antibodies; Bioworld Technology, St Louis Park, MN, USA), anti-p-38 (1:1,000, rabbit polyclonal antibodies; Cell Signaling Technology, Inc., Danvers, MA, USA), and anti- $\beta$-actin (1:1,000, a mouse polyclonal antibody), (Santa Cruz Biotechnology, Inc., Dallas, TX, USA) at $4^{\circ} \mathrm{C}$ overnight, washed with Tris-buffered saline and incubated with a horseradish peroxidase-conjugated antirabbit immunoglobulin (Ig)G/antimouse IgG secondary antibody at $37^{\circ} \mathrm{C}$ for 1 hour. The antibody-bound proteins were detected using an enhanced chemiluminescence reagent (EMD Millipore, Billerica, MA, USA). Quantification of the Western blot and electrophoretic mobility shift assay (EMSA) data were performed by measuring the intensity of the band using the Image J analysis program (Image J, NIH).

\section{Electrophoretic mobility shift assay (EMSA)}

EMSA was used to assess the NF- $\kappa B$ activation of HUVECs. Briefly, following the incubation periods, HUVEC nuclear extracts were prepared using Nuclear and Cytoplasmic Extraction Reagents (Thermo Fisher Scientific). The protein concentration of each nuclear extract was quantified using the bicinchoninic acid protein assay. Ten micrograms of nuclear protein was incubated in binding buffer containing $50 \mathrm{ng} / \mu \mathrm{L}$ of poly $(\mathrm{dI} \cdot \mathrm{dC}), 2.5 \%$ glycerol, $0.05 \% \mathrm{NP}-40,5 \mathrm{mM}$ of $\mathrm{MgCl}_{2}$, and $20 \mathrm{fmol}$ of Biotin end-labeled oligonucleotides at room temperature for 20 minutes. The labeled oligonucleotides had the following sequences: 5'-AGT TGA GGG GAC TTT CCC AGG C-3' and 5'-GCC TGG GAAAGT CCC CTCAAC T-3'. Proteindeoxyribonucleic acid (DNA) complexes were separated from the free DNA probe via electrophoresis through $4 \%$ native polyacrylamide gels. Gels were dried, and protein-DNA complexes were then visualized using enhanced chemiluminescence.

\section{Statistical analysis}

The data are expressed as the means \pm standard deviation or means \pm standard error of the mean (SEM). Statistical comparisons of the means were performed using one-way analysis of variance with SAS 6.12 software (SAS Institute Inc., Cary, NC, USA). The differences were considered to be significant when the $P$-value was $<0.05$.

\section{Results \\ Characterization and dispersion of HANPs}

In this study, we investigated the main physicochemical properties of our synthetic HANPs. TEM analyses revealed that the HANPs were near spherical, or they exhibited slightly elongated shapes (an average of $15 \mathrm{~nm}$ in diameter). The size and morphology of HANPs are illustrated in Figure 1A and B. The XRD pattern of the HANPs showed that the structure of the prepared sample was similar to that of the HA standard, as shown in Figure 1C. Our data of the HANPs are highly consistent with the standard HA database (powder diffraction file no 09-0432), indicating that the chief inorganic phase of our synthetic NPs is an HA crystal. Furthermore, our energy-dispersive X-ray spectroscopy data show that the $\mathrm{Ca} / \mathrm{P}$ ratio in our samples is close to 1.67 which is similar to the theoretical value in HA, suggesting our synthetic HAP is of high purity (Figure 1D). Surface charge and hydrodynamic diameter are two important properties of NP dispersions in aqueous systems. For the remainder of the study, NPs were characterized in complete cell culture medium (1:1 mixture of EC medium and RPMI 1640 medium). The hydrodynamic size of the NPs, as measured by dynamic light scattering, was approximately $248 \mathrm{~nm}$, revealing that the NPs were well dispersed in the cell culture medium (Table 1). The zeta-potential of the NPs was determined and displayed a positive potential $(-8.89 \mathrm{mV})$ in cell culture medium, indicating that the NPs presented a negative surface charge. Additionally, to examine the surface property of the HANPs, we estimated the Brunauer-Emmett-Tellerspecific surface area. As shown in Table 1, the specific surface area of our prepared HANPs was $109 \mathrm{~m}^{2} / \mathrm{g}$, which was significantly larger than the commercial HANPs measured by other researchers $\left(7.4 \mathrm{~m}^{2} / \mathrm{g}\right),{ }^{15}$ indicating that the HANPs have a higher biological reactivity. ${ }^{21-23}$

\section{Effects of direct HANP exposure on HUVECs and THP-I cell viability}

To determine the effects of HANPs on HUVEC and THP-1 cell viability in vitro, mitochondrial function was measured using the MTS assay after culturing with serial dilutions $(25 \mu \mathrm{g} / \mathrm{mL}, 50 \mu \mathrm{g} / \mathrm{mL}, 100 \mu \mathrm{g} / \mathrm{mL}, 200 \mu \mathrm{g} / \mathrm{mL}$, and $400 \mu \mathrm{g} / \mathrm{mL}$ ) of HANPs for 24 hours. As depicted in Figure 2, both HUVEC and THP-1 cell viability decreased in relation 

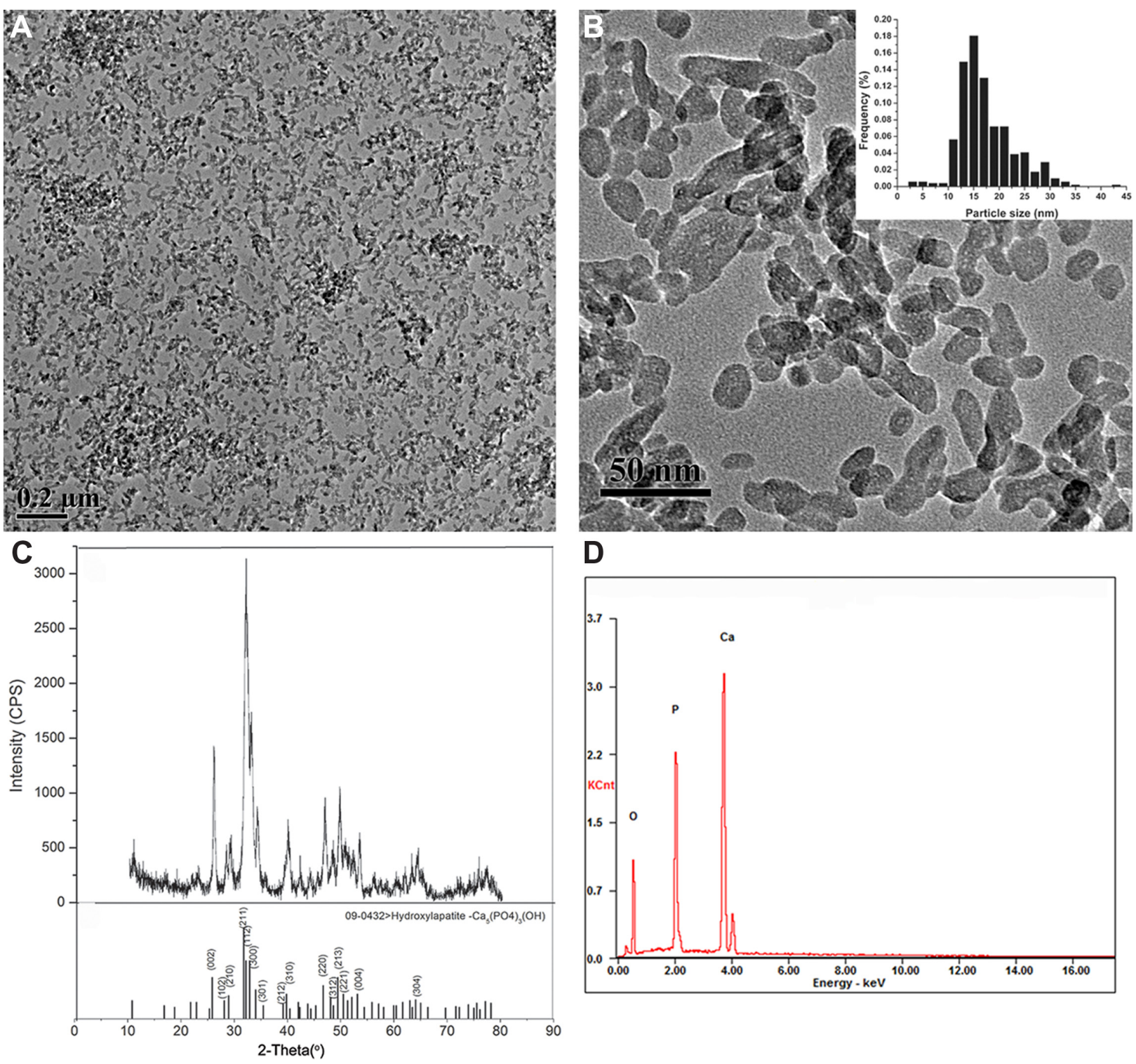

Figure I Characterization of hydroxyapatite nanoparticles.

Notes: (A and B) Transmission electron microscopy image; inset: histogram showing size distribution; about 500 NPs were considered for the sample to obtain the size distribution histogram. (C) X-ray diffraction patterns. (D) Energy-dispersive X-ray spectroscopy spectra.

Abbreviations: CPS, counts per second; KCnt, I,000 counts; NPs, nanoparticles.

to increasing concentrations of HANPs. At concentrations of $100 \mu \mathrm{g} / \mathrm{mL}$ or below, no significant differences were observed in the cell viability between HANP-stimulated cells and untreated cells $(P>0.05)$. After treatment with HANPs at $200 \mu \mathrm{g} / \mathrm{mL}$ and $400 \mu \mathrm{g} / \mathrm{mL}$, HUVEC viability dramatically decreased to $65 \%$ and $59 \%$, respectively. Similarly, at $200 \mu \mathrm{g} / \mathrm{mL}$ and $400 \mu \mathrm{g} / \mathrm{mL}$, HANPs also caused a significant inhibition of mitochondrial function in THP-1 cells $(P<0.05)$.

\section{Cellular uptake and localization of HANPs}

It is known that cellular uptake is not only a critical step in gene carrier or intracellular drug delivery, but it is also directly related to the biological impact of NPs. To follow

Table I Characterization of the particle parameters of HANPs

\begin{tabular}{llllll}
\hline Material & Morphology & $\begin{array}{l}\text { Average diameter } \\
\text { by TEM }(\mathbf{n m})\end{array}$ & $\begin{array}{l}\text { Hydrodynamic } \\
\text { size }(\mathbf{n m})\end{array}$ & $\begin{array}{l}\text { Surface } \\
\text { area }\left(\mathbf{m}^{2} / \mathbf{g}\right)\end{array}$ & $\begin{array}{l}\text { Zeta-potential } \\
(\mathbf{m V})\end{array}$ \\
\hline HANPs & Near spherical & $15 \pm 5$ & $248 \pm 33$ & $109 \pm 0.34$ & $-8.89 \pm 0.04$ \\
\hline
\end{tabular}

Abbreviations: HANPs, hydroxyapatite nanoparticles; TEM, transmission electron microscopy. 


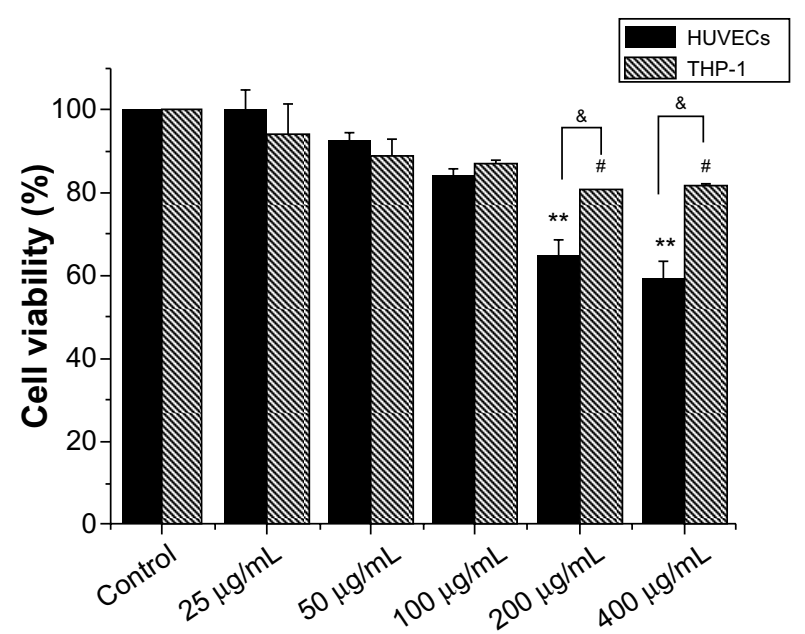

Figure 2 Cytotoxicity of hydroxyapatite nanoparticles to HUVEC and THP-I cells. Notes: Cells were exposed to increasing doses of hydroxyapatite nanoparticles for 24 hours, and cytotoxicity was determined by the MTS assay. Normal HUVECs or THP-I cells without nanoparticle treatment served as control. Results are presented in mean \pm standard error of the mean of the three independent experiments; each was carried out in triplicate. ${ }^{\sharp} P<0.05$; ${ }^{* * P}<0.0$ I versus control; ${ }^{\&} P<0.05$ significant difference between the compared groups.

Abbreviations: HUVECs, human umbilical vein endothelial cells; MTS, 3-(4, 5-dimethylthiazol-2-yl)-5-(3-carboxymethoxyphenyl)-2-(4-sulfophenyl)-2Htetrazolium.

the internalization and intracellular localization of HANPs, HUVECs and THP-1 cells were exposed to the HANPs at a low toxic dose $(100 \mu \mathrm{g} / \mathrm{mL})$ and were then examined using TEM. As shown in Figure 3, most of the HANPs in HUVECs and THP-1 cells were confined to vesicles and lysosomes within the cytoplasm and did not enter the nucleus. Moreover, based on the imaging, HUVECs retained their cellular morphology without damage following the uptake of HANPs. In contrast, in HANP-stimulated THP-1 cells, several pseudopodia and large vacuoles appeared, indicating the activated state of the cell.

\section{Cytokine production by HANPs in monoculture and cocultures}

To obtain a more realistic assessment of the effects of HANPs on the human vascular system, this study simulated the human vasculature using a coculture model composed of ECs and monocytes. A proinflammatory response was first investigated after the cocultures were exposed to HANPs for 24 hours. Responses were compared with those of HUVECs or monocyte monocultures at the same condition. Four patterns of cytokine release (TNF- $\alpha$, IL-1 $\beta$, IL-6, and IL-8) were observed. As shown in Figure 4A and B, THP-1 cells exposed to HANPs exhibited significant increases in TNF- $\alpha$ (up to 15-fold) and IL-1 $\beta$ (up to twofold) in both monoculture and cocultures; however, cocultures with HUVECs had no appreciable effects on the levels of TNF- $\alpha$ and IL- $1 \beta$ compared with monocultures stimulated with HANPs $(P>0.05)$. Moreover, in HUVECs exposed to HANPs, no relevant increases were observed for any cytokine, whereas in the cocultures of HUVECs/THP-1 cells with HANPs, a strong increase of IL-6 production was induced (Figure 4C and D).

To clarify whether cell-derived soluble factors induced by HANPs are responsible for secondary proinflammatory cytokine release in cocultures, HUVECs and THP-1 cells were pretreated with HANPs for 24 hours, and the cell-free supernatant was collected to stimulate another cell type for 24 hours. As shown in Figure 4, the supernatants of HANP-stimulated THP-1 cells exhibited a significant increase in IL-6 release from HUVECs, whereas the supernatants of HANP-stimulated HUVECs revealed no effects on TNF- $\alpha$ and IL- $1 \beta$ release from the THP-1 cells, suggesting that HANPs might cause an indirect activated effect on HUVECs by monocyte-derived soluble factors.

\section{Endothelial adhesion molecule expression induced by HANPs in monoculture and cocultures}

To further evaluate EC activation in HANP-stimulated cocultures, endothelial adhesion molecule expression (CD106, CD54, and CD62E) was measured using flow cytometry. As shown in Figure 5, HANPs alone did not induce CD54, CD106, or CD62E expression; however, when cocultured with THP-1 cells, HANPs significantly increased the percentage of HUVECs that expressed CD54, CD106, and CDD62E. In addition, the supernatants of HANP-stimulated monocytes also increased the expression of CD54, CD106, and CD62E. To investigate whether HUVEC monolayers in HANP-stimulated coculture would become more adherent for leukocytes, flow cytometry was performed to examine the $v W F$ expression of HUVECs. As shown in Figure 5C, the vWF expression of separated HUVECs from the HANP-treated coculture was decreased to $91.9 \% \pm 2.12 \%$, slightly lower than the untreated HUVECs $(99.2 \% \pm 0.69 \%)$ $(P<0.05)$, indicating that there might be some monocyte adhesion to HUVECs.

\section{Involvement of $\mathrm{p} 38$, JNK, and NF- $\mathrm{KB}$ in the activation of HUVECs in HANP- stimulated cocultures}

To clarify the possible signaling pathway of the proinflammatory effects of HANPs on HUVECs in coculture, the activation levels of JNK, p38, and NF- $\mathrm{KB}$ were 

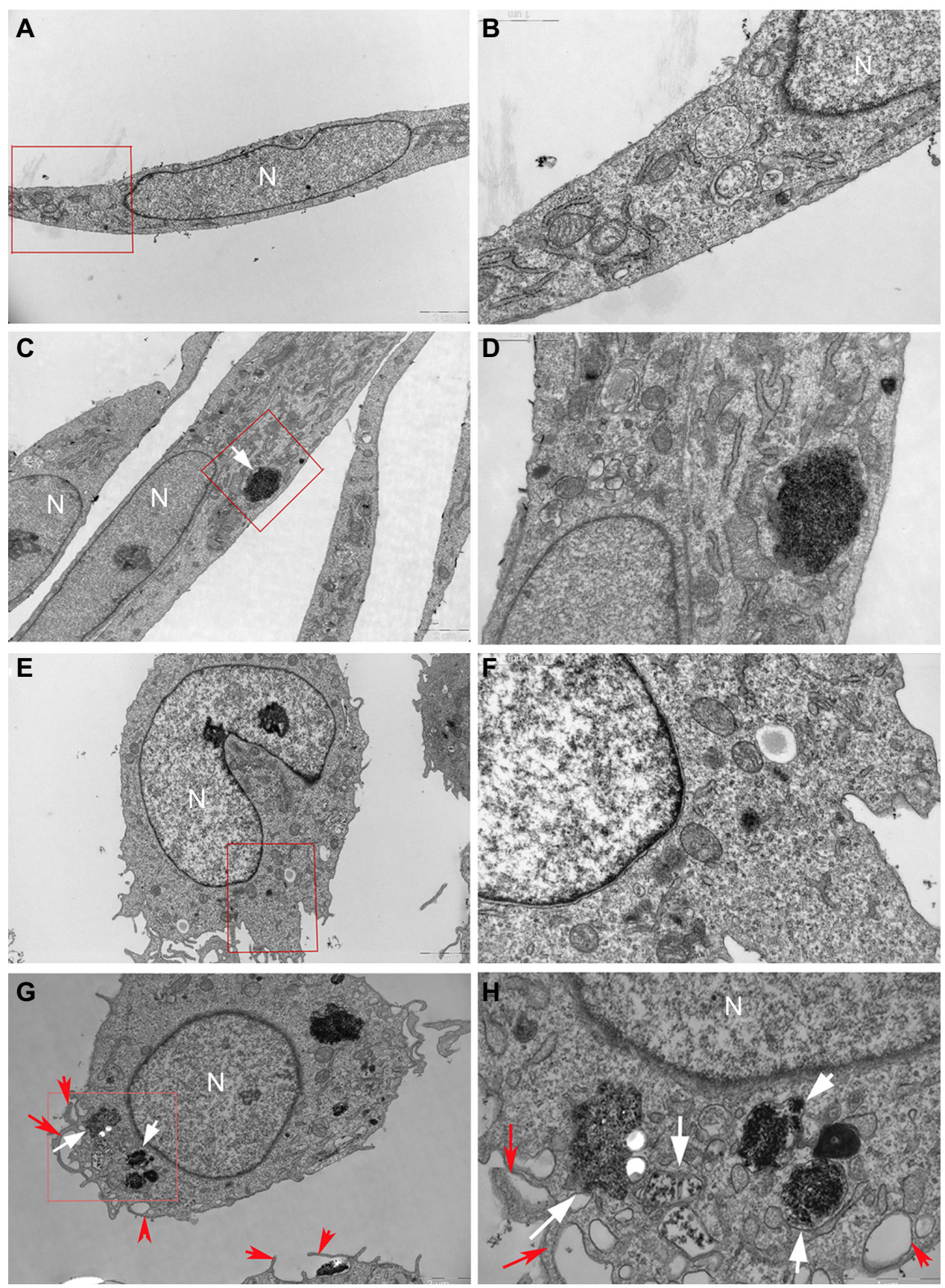

Figure 3 Uptake of HANPs by HUVEC and THP-I cells.

Notes: TEM micrographs of cells exposed for 24 hours to HANPs. (A and B) HUVECs without any treatment; (C and D) HUVECs treated with HANPs; (E and F) THP-I cells without any treatment; ( $\mathbf{G}$ and $\mathbf{H})$ THP-I cells treated with HANPs. (A, C, E and $\mathbf{G}$ ) Overall cell morphology (scale bar: $2 \mu \mathrm{m})$. (B, D, F and $\mathbf{H})$ Higher magnification of cells in red boxed areas (scale bar: $1 \mu \mathrm{m}$ ). White arrows denote nanoparticles. Red arrows indicate the protrusion of the plasma membrane for phagocytosis.

Abbreviations: N, nucleus; HANPs, hydroxyapatite nanoparticles; HUVECs, human umbilical vein endothelial cells; TEM, transmission electron microscopy.

detected in HANP-stimulated cocultures. As depicted in Figure 6A, the cocultures had significantly elevated JNK and p38 MAPK phosphorylation levels after treatment with HANPs when compared with ECs cultured alone. Similarly, NF- $\kappa B$ was significantly activated in HANPstimulated HUVEC cocultures compared with HUVECs in monoculture (Figure 6B).

\section{Discussion}

Recently, more efforts have been focused on the possibility of utilizing synthetic HANPs for multiple applications, such as magnetic resonance imaging, antimicrobial treatments, drug delivery, and gene transfection; however, their biocompatibility and toxicity remain unclear. ${ }^{3,6} \mathrm{~A}$ major limitation of in vitro cell models in investigations of NP-induced biological 

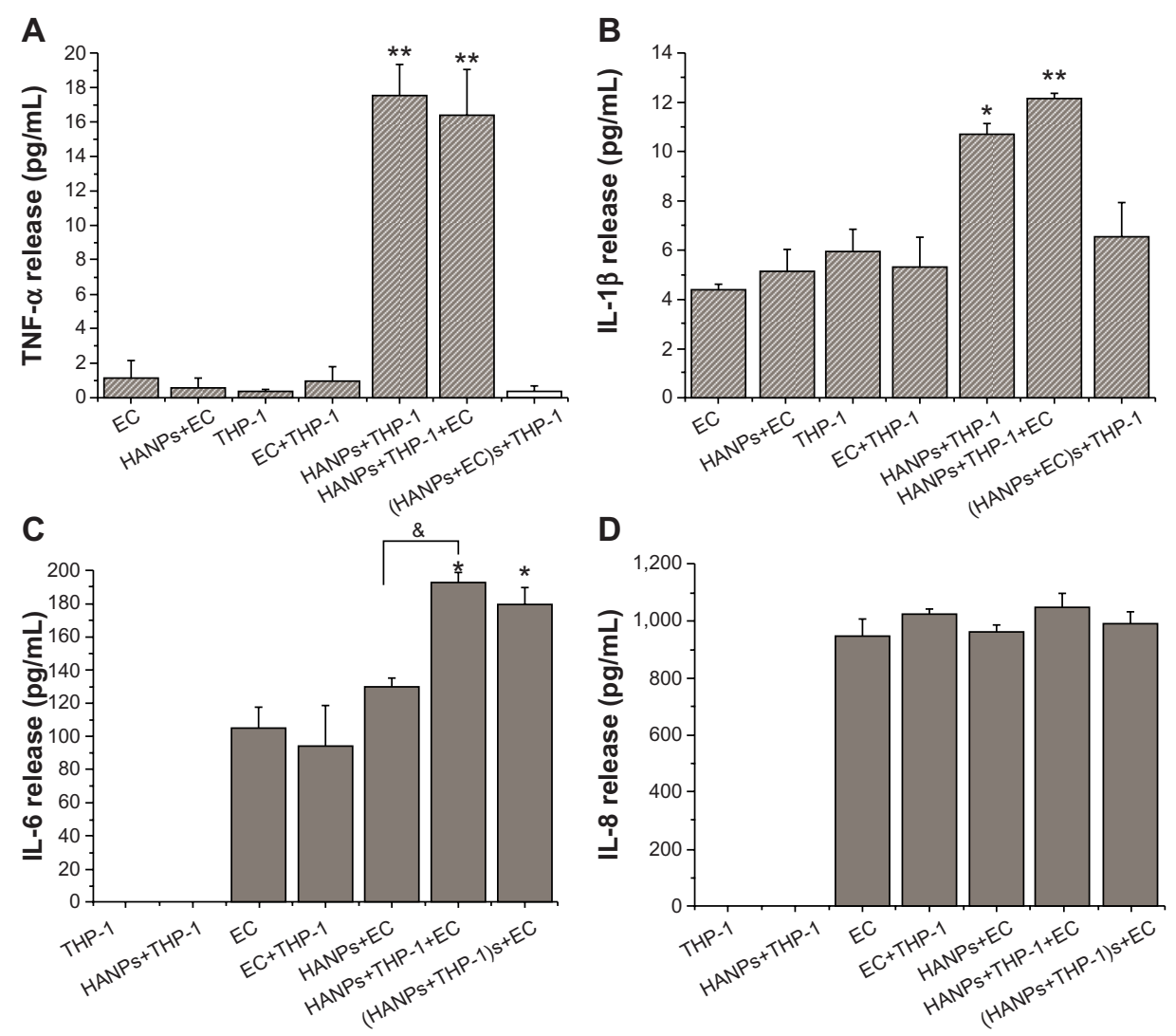

Figure 4 Proinflammatory cytokine production induced by HANPs in mono- and cocultures.

Notes: (A) TNF- $\alpha$ release; (B) IL-I $\beta$ release; (C) IL-6 release; (D) IL-8 release. Normal HUVECs or THP-I cells or cocultures without NPs treatment served as control. Results are presented as the mean \pm standard error of the mean of three independent experiments, each of which was carried out in triplicate. $* P<0.05$; $* * P<0.0$ I versus control; ${ }^{2} P<0.05$ significant difference between compared groups.

Abbreviations: TNF- $\alpha$, tumor necrosis factor-alpha; EC, endothelial cells; HANPs, hydroxyapatite nanoparticles; (HANPs+THP-I)s+EC, human umbilical vein endothelial cells treated with supernatants of hydroxyapatite nanoparticle-stimulated THP-I cells; (HANPs+ECs)+THP-I, THP-I cells treated with the supernatants of hydroxyapatite nanoparticle-stimulated human umbilical vein endothelial cells; IL, interleukin; NPs, nanoparticles.

effects is that common monoculture systems do not represent a realistic model of how NPs will interact with a specific organ of the body due to the lack of cellular interactions and extracellular signal communications that are important in an in vivo situation. ${ }^{24}$ The present study using coculture models of ECs and monocytes with HANPs to mimic the complex physiological functionality of the vascular system demonstrate that monocytes could play an important role in the mechanisms of endothelium dysfunction induced by HANP exposure. According to our findings, at a subcytotoxic dose, direct HANP exposure did not cause EC injury, but when cocultured with THP-1 cells, we observed a significant inflammatory activation of HUVECs. This process is possibly dependent on the action of monocyte-derived soluble factors via $\mathrm{p} 38 / \mathrm{JNK}$ MAPK and NF- $\mathrm{KB}$ activation. Thus, in the present study, we observed a series of cellular and molecular interactions initiated by HANPs between monocytes and ECs that resulted in an inflammatory cascade through a complex network of pathways.
In this study, HUVECs and THP-1 cells were used to evaluate the cytotoxic effect of HANPs by the MTS assay. ECs and monocytes were selected because NPs intravenously administered into the systemic circulation are the most common route of exposure to nanomedicine. At concentrations of $200 \mu \mathrm{g} / \mathrm{mL}$ and greater, HANPs became cytotoxic in both cell types (Figure 2). Exposure to a subcytotoxic dose can help identify potential health effects of NPs other than those due to cytotoxicity; ${ }^{25}$ thus, we chose to work with a lower particle concentration in the following studies $(100 \mu \mathrm{g} / \mathrm{mL})$. Under these conditions, neither cell type exhibited dramatic cell death after a 24-hour treatment with NPs. Given that it has been demonstrated that the cellular uptake of NPs plays a pivotal role in the alteration of various cellular functions, such as cell viability and the inflammatory response, ${ }^{26}$ the internalization and intracellular localization of HANPs were subsequently examined by TEM. In this assay, different cellular uptake behaviors were observed in HUVECs and THP-1 cells after treatment with HANPs 

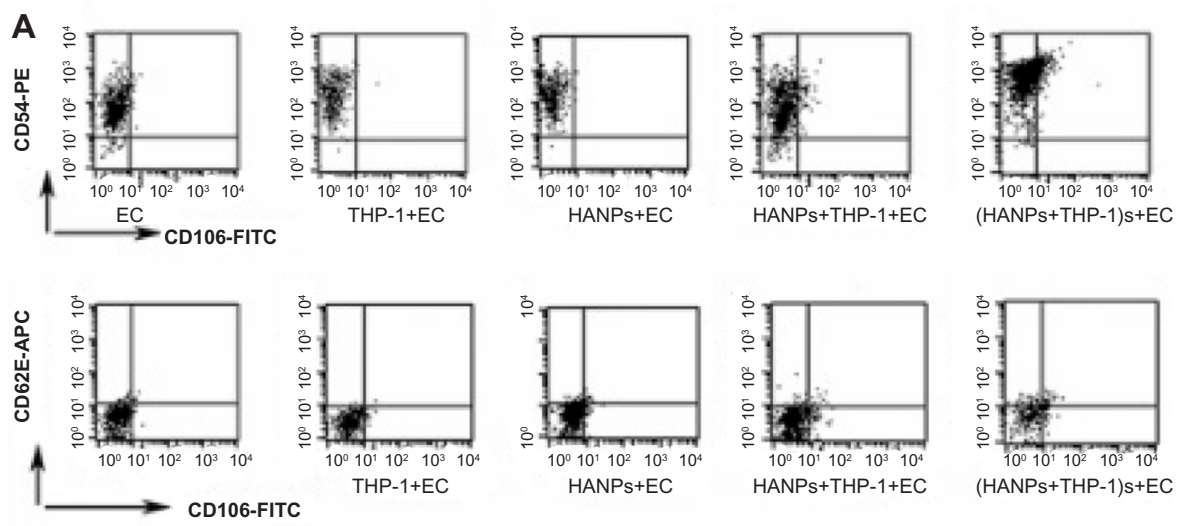

B

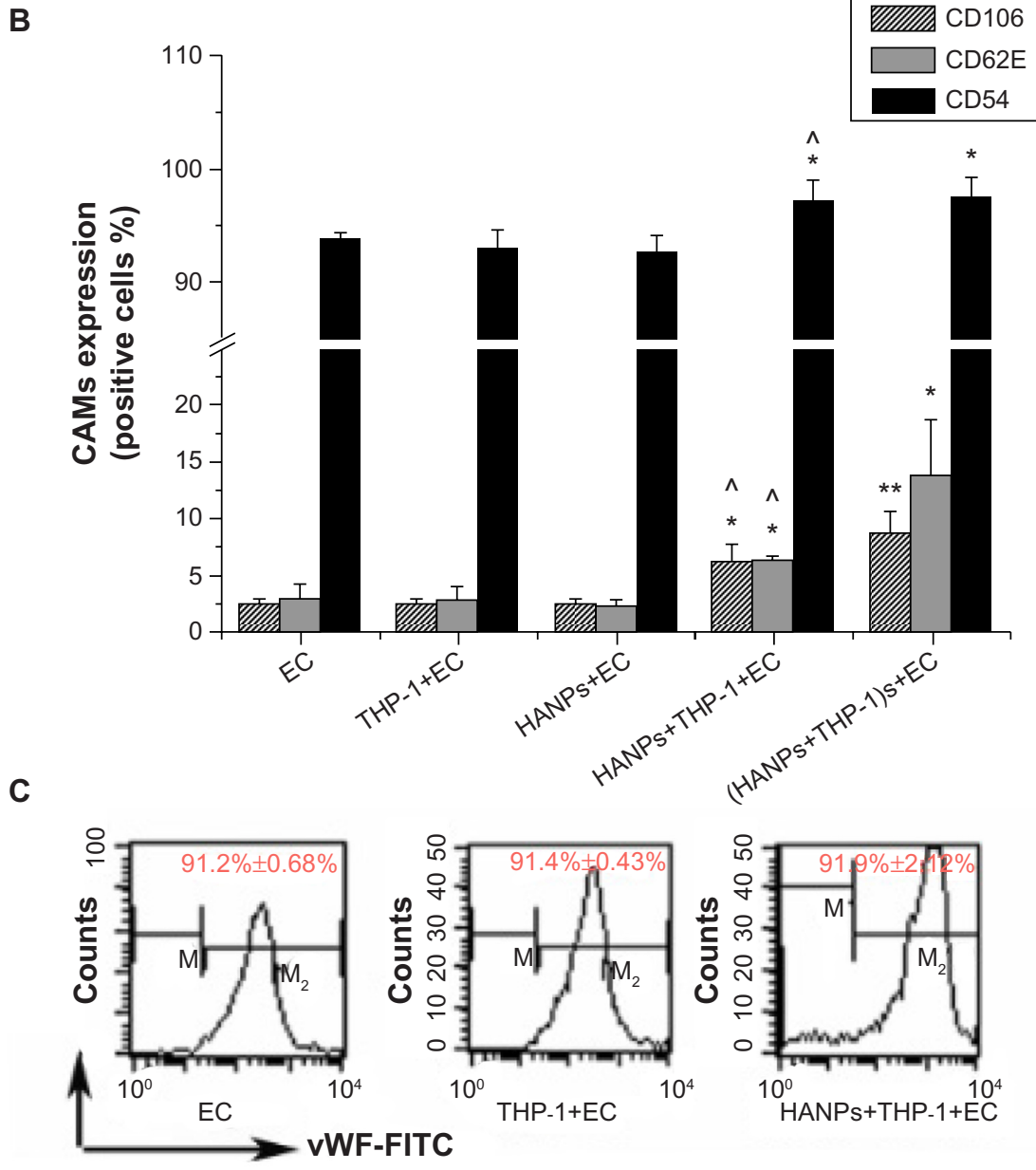

Figure 5 CAMs expression induced by HANPs in HUVECs in mono- and cocultures.

Notes: (A) Dot-plot of flow cytometry analysis showing the expressions of CD54, CDI06, and CD62E on HUVECs in mono-and cocultures exposed to HANPs for 24 hours. (B) Bar graph shows the percentage of CD54-, CDI06-, and CD62E-positive cells in NP-stimulated HUVECs in monoculture or in coculture. (C) The vWF expression on HUVECs in HANP-stimulated cocultures. Normal HUVECs served as the negative control. Data represent the means \pm standard error of the means; $\mathrm{n}=3$. $* P<0.05 ; * * P<0.01$ versus control; ${ }^{\wedge} P<0.05$ versus HUVECs with HANPs.

Abbreviations: $C D$, cluster of differentiation; EC, endothelial cells; FITC, fluorescein isothiocyanate isomer I; HANPs, hydroxyapatite nanoparticles; vWF, von Willebrand factor; CAMs, cell adhesion molecules; HUVECs, human umbilical vein endothelial cells; NP, nanoparticle; n, number.

for 24 hours (Figure 3). In both cell lines, the HANPs were tightly packed and largely confined to spherical vesicles resembling lysosomes within the cytoplasm. Importantly, after HANP uptake, HUVECs did not display a change of morphology (Figure 3C-D). In contrast, a prominent change in THP-1 cells was observed, which included increased phagocytic activity, as demonstrated by large vacuoles and multiple pseudopodia of the plasma membrane, suggesting the potential immune activation of these particular cells (Figure 3G-H). 


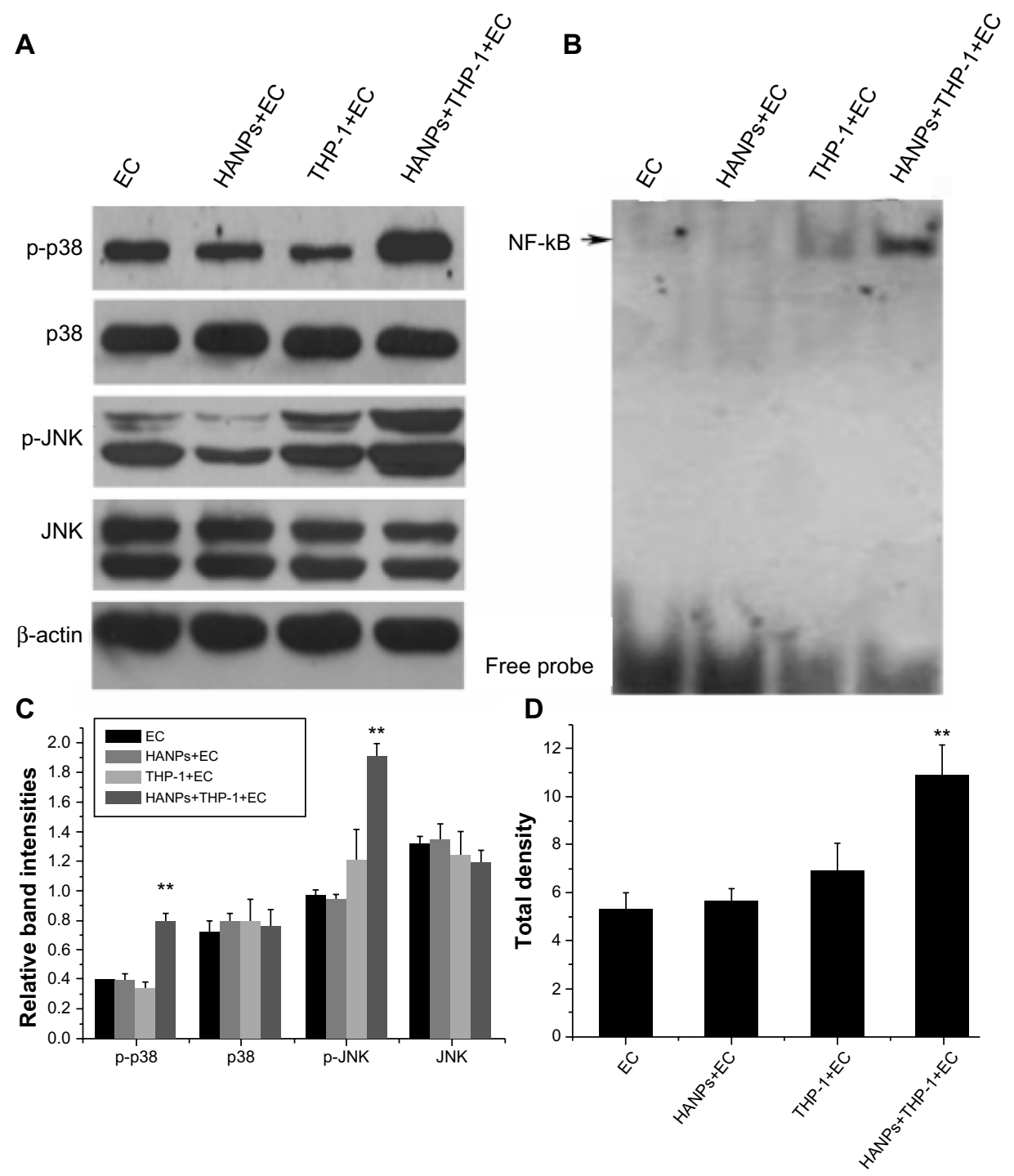

Figure 6 The activation of JNK, p38, and NF-אB by HANPs in HUVECs in mono- and cocultures.

Notes: (A) The activation of JNK and p38 by HANPs in HUVECs in mono- and cocultures. Aliquots of cell lysates were separated by SDS-PAGE and analyzed for protein expression by Western blotting, as described in section 2. $\beta$-actin was used as an internal control to monitor for equal loading. (B) The activation of NF-KB by HANPs in HUVECs in mono- and cocultures. (C) The relative density of the bands normalized to beta-actin by gray value analysis ( $\beta$-actin as control); (D) The total density of

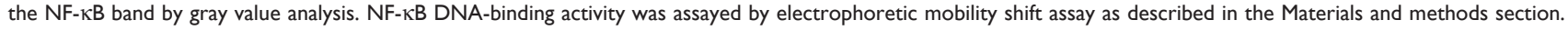
**Represents a statistically significant difference from EC $(P<0.01)$.

Abbreviations: JNK, c-Jun N-terminal kinase; EC, endothelial cells; HANPs, hydroxyapatite nanoparticles; NF- KB, nuclear factor-kappa B; HUVECs, human umbilical vein endothelial cells; SDS-Page, sodium dodecyl sulfate polyacrylamide gel electrophoresis.

Cytokines are important regulators of immune, inflammatory, and vascular reactions, and they play critical roles in cardiovascular disease. ${ }^{27}$ Among these cytokines, TNF- $\alpha$ and IL- $1 \beta$ are generally markers of monocyte activation. Moreover, IL-6 is clinically considered a biomarker of endothelial dysfunction and an independent risk factor for atherosclerosis. ${ }^{28,29}$ As a next step, to further investigate the impact of NP uptake on the cell activation status, we measured the concentrations of TNF- $\alpha$, IL- $1 \beta$, IL- 6 , and IL-8 present in HUVECs and THP-1 cell supernatants after direct exposure to HANPs. These experiments demonstrated that increased levels of TNF- $\alpha$ and IL- $1 \beta$ were released in
THP-1 cells after treatment with HANPs (Figure 4A and B), whereas no proinflammatory cytokines were released in HUVECs alone (Figure 4C and D).

Since the interaction of the monocytes and ECs that line the blood vessels is a critical process in vascular homeostasis and in pathological conditions of inflammation or thrombosis, the monocyte-EC coculture model is frequently used to assess the biocompatibility of synthetic materials, such as vascular grafts, artificial hearts, and coronary-artery stents for vascular application. ${ }^{30-34}$ Recently, some researchers have attempted to use an in vitro coculture model of immune cells and ECs to evaluate the inflammatory potential of 
nanomaterials, and they found that exposure to these NPs (for example, titanium dioxide $\left[\mathrm{TiO}_{2}\right]$ or silicone dioxide $\left.\left[\mathrm{SiO}_{2}\right]\right)$ in direct-contact cocultures led to dramatically elevated levels of proinflammatory cytokines. ${ }^{10,35}$ Here, we found that the coculture of HUVEC/THP-1 cells resulted in an approximately twofold increase in IL-6 concentrations compared with the individual cell types alone (Figure 4C). Separating the two cell types and treating HUVECs with conditioned media from HANP-exposed THP-1 cells also significantly increased the release of IL-6, indicating that the induction of IL-6 in HUVECs may be largely dependent on the HANP-activated monocytes (Figure 4C). Previous studies have reported that noncontact cocultures of ECs and macrophages/monocytes strongly induced more potent inflammatory cytokine release in ECs upon silica particle exposure compared with monocultures alone. ${ }^{36,37}$ However, coculture or treatment with the supernatants from HANP-stimulated HUVECs did not increase the levels of TNF- $\alpha$ and IL-1 $\beta$ in THP- 1 cells when compared with the THP-1 cells directly exposed to HANPs, indicating that the HANP-stimulated ECs were relatively ineffective on monocyte activation (Figure 4A and B).

When undergoing activation, apart from IL-6, ECs also express various CAMs, such as ICAM-1 (CD54), VCAM-1 (CD106), and E-selectins (CD62E). Generally, ICAM-1 is constitutively present in ECs, and its expression is increased by proinflammatory stimuli. ${ }^{38} \mathrm{VCAM}-1$ and E-selectin are not routinely expressed under physiologic conditions, but they can be induced in activated endothelium. The expression of VCAM-1 and E-selectin is important in the initial steps of monocyte recruitment to atherosclerotic lesions and in the rolling of monocytes on the endothelial surface, whereas ICAM-1 is involved in the firm adhesion step in leukocyte infiltration. ${ }^{39,40}$ CAM expression and established markers of endothelial dysfunction have been clinically considered to be associated with cardiovascular risk factors, and they may predict the development of cardiovascular disease. ${ }^{41}$ Thus, to further verify the contribution of monocytes in EC activation induced by HANP exposure, CAM expression in HUVECs was measured using flow cytometry. Consistent with proinflammatory cytokine results after exposure to HANPs, cocultures of ECs and monocytes had significantly greater increased levels of VCAM-1, E-selectin and ICAM-1 expression compared with HUVECs cultured alone. vWF is considered to be a classic marker of ECs, because it has been found only in ECs, megakaryocytes, and platelets. ${ }^{42}$ Thus, we assessed the THP-1 cells' adhesion to HUVECs by cell-specific surface markers (vWF). Our data showed that the vWF expression of separated HUVECs from the HANP-treated coculture was slightly lower than that of the HUVECs from the untreated coculture $(P<0.05)$, indicating that HANPs may elicit an attachment of monocytes to HUVECs. Additionally, HUVECs incubated with supernatants of HANP-stimulated monocytes expressed levels of VCAM-1, E-selectin, and ICAM-1 that were similar to those generated by the direct-contact coculture system, suggesting that monocyte-derived soluble factors were responsible for the activation of ECs (Figure 5). Among the soluble mediators possibly involved, TNF- $\alpha$ and IL-1 $\beta$, which are primarily produced by monocytes and are wellknown inducers of EC activation,were good candidates. ${ }^{43}$ Taken together, these results suggest that direct exposure to HANPs could result in TNF- $\alpha$ and IL-1 $\beta$ production in THP-1 cells and that both cytokines, once released, might stimulate IL-6 generation and CAM expression by HUVECs. These results were consistent with those from previous studies that demonstrated that the release of TNF- $\alpha$ and IL- $1 \beta$ from particle-activated monocytes/macrophages might be the critical determinants for increased synthesis of IL-6 or CAM expression in ECs. ${ }^{7,9,36}$

It is a widely accepted view that intracellular MAPK signaling cascades probably play an important role in the pathogenesis of cardiovascular disease. ${ }^{39}$ To clarify the possible signaling pathway of the proinflammatory effects of HANPs on HUVECs in coculture, we detected the activation levels of JNK, p38, and NF-кB. p38 and JNK belong to the MAPK superfamily and are stress-activated serine/threonine protein kinases with major functions in apoptosis, cytokine production, transcriptional regulation, and inflammation. ${ }^{44}$ Various inflammatory cytokines are capable of activating p38 and JNK MAPK, which has been shown to regulate inflammation and induce endothelial dysfunction. ${ }^{45,46}$ In addition, NF- $\kappa$ B is also a critical transcription factor involved in the transcription of proinflammatory cytokines and the expression of CAMs, such as E-selectin, VCAM-1, and ICAM-1. ${ }^{47,48}$ Our results clearly demonstrated that HANPs alone could not induce JNK, p38, or NF- $\kappa \mathrm{B}$ activation in HUVECs; however, HANP-stimulated cocultures significantly elevated the phosphorylation levels of $\mathrm{p} 38 / \mathrm{JNK}$ MAPK and the activity of NF- $\kappa \mathrm{B}$, suggesting that the activation of $\mathrm{JNK}, \mathrm{p} 38$, and $\mathrm{NF}-\kappa \mathrm{B}$ in ECs by exposure to HANPs required the involvement of monocytes. It has been demonstrated that IL-1 $\beta$ mainly induces proinflammatory cytokine release, such as IL-6 gene expression in HUVECs, which is a $\mathrm{p} 38 / \mathrm{JNK}$-dependent process. ${ }^{49}$ However, TNF- $\alpha$ more profoundly affected the expression of CAMs via the NF- $\kappa$ B pathway. ${ }^{49}$ TNF- $\alpha$ and IL- $1 \beta$ release by HANP-stimulated monocytes may induce p38/JNK MAPK and NF- $\kappa B$ activation in HUVECs, suggesting that HANPs can indirectly induce ECs' inflammatory response by activating monocytes. However, in this study, although we have found that HANPs 
could directly induce TNF- $\alpha$ and IL- $1 \beta$ release from monocytes, and that these cytokines released by HANP-stimulated monocytes play a critical role in monocyte-mediated inflammatory activation in HUVECs, we cannot rule out other potential proinflammatory mechanisms of HANP-activated monocytes against HUVECs. The detailed mechanism of this phenomenon needs to be further investigated.

\section{Conclusion}

In summary, our data provide evidence that HANPs could be taken up by both monocytes and ECs, and that HANP phagocytosis caused an inflammatory response in monocytes, but not in ECs. In addition, although HANPs had no direct effect on endothelial inflammation, we determined that HANPs induced an indirect activation of ECs, resulting in increased IL-6 production and elevated adhesion molecule expression after coculture with monocytes. The potential proinflammatory effect of HANPs is primarily mediated by the release of soluble factors from activated monocytes, which leads to an inflammatory response of the endothelium that is possibly dependent on p38/ JNK MAPK and NF- $\kappa \mathrm{B}$ signaling activation. Thus, the use of the in vitro monocyte-EC coculture model on the biocompatibility assessment of HANPs could reveal their potential proinflammatory effects on ECs, suggesting that exposure to HANPs could increase the risk of cardiovascular disease.

\section{Acknowledgments}

This work was supported by grants from the National Natural Science Foundation of China (no 81271700, no 81201191, no 31070843 ), and the Shanghai Sci-Tech Committee Foundation (13DZ2291100).

\section{Disclosure}

The authors report no conflicts of interest in this work.

\section{References}

1. Aulakh TS, Jayasekera N, Kuiper JH, Richardson JB. Long-term clinical outcomes following the use of synthetic hydroxyapatite and bone graft in impaction in revision hip arthroplasty. Biomaterials. 2009;30(9):1732-1738.

2. Zhou H, Lee J. Nanoscale hydroxyapatite particles for bone tissue engineering. Acta Biomater. 2011;7(7):2769-2781.

3. Fox K, Tran PA, Tran N. Recent advances in research applications of nanophase hydroxyapatite. Chemphyschem. 2012;13(10):2495-2506.

4. Kadono $\mathrm{H}$, Furuzono T, Masuda $M$, et al. In vivo evaluation of hydroxyapatite nanocoating on polyester artificial vascular grafts and possibility as soft-tissue compatible material. ASAIO J. 2010;56(1):61-66.

5. Ashokan A, Chandran P, Sadanandan AR, et al. Development and haematotoxicological evaluation of doped hydroxyapatite based multimodal nanocontrast agent for near-infrared, magnetic resonance and X-ray contrast imaging. Nanotoxicology. 2012;6(6):652-666.
6. Uskoković V, Uskoković DP. Nanosized hydroxyapatite and other calcium phosphates: chemistry of formation and application as drug and gene delivery agents. J Biomed Mater Res B Appl Biomater. 2011;96(1): 152-191.

7. Donaldson KI, Duffin R, Langrish JP, et al. Nanoparticles and the cardiovascular system: a critical review. Nanomedicine (Lond). 2013;8(3):403-423.

8. Kristovich R, Knight DA, Long JF, Williams MV, Dutta PK, Waldman WJ. Macrophage-mediated endothelial inflammatory responses to airborne particulates: impact of particulate physicochemical properties. Chem Res Toxicol. 2004;17(10):1303-1312.

9. Weldy CS, Wilkerson HW, Larson TV, Stewart JA, Kavanagh TJ. DIESEL particulate exposed macrophages alter endothelial cell expression of eNOS, iNOS, MCP1, and glutathione synthesis genes. Toxicol In Vitro. 2011;25(8):2064-2073.

10. Shaw CA, Robertson S, Miller MR, et al. Diesel exhaust particulate exposed macrophages cause marked endothelial cell activation. Am J Respir Cell Mol Biol. 2011;44(6):840-851.

11. Liu X, Xue Y, Ding T, Sun J. Enhancement of proinflammatory and procoagulant responses to silica particles by monocyte-endothelial cell interactions. Part Fibre Toxicol. 2012;9:36.

12. Nadra I, Boccaccini AR, Philippidis P, et al. Effect of particle size on hydroxyapatite crystal-induced tumor necrosis factor alpha secretion by macrophages. Atherosclerosis. 2008;196(1):98-105.

13. Scheel J, Weimans S, Thiemann A, Heisler E, Hermann M. Exposure of the murine RAW 264.7 macrophage cell line to hydroxyapatite dispersions of various composition and morphology: assessment of cytotoxicity, activation and stress response. Toxicol In Vitro. 2009;23(3):531-538.

14. Grandjean-Laquerriere A, Tabary O, Jacquot J, et al. Involvement of tolllike receptor 4 in the inflammatory reaction induced by hydroxyapatite particles. Biomaterials. 2007;28(3):400-404.

15. Motskin M, Wright DM, Muller K, et al. Hydroxyapatite nano and microparticles: correlation of particle properties with cytotoxicity and biostability. Biomaterials. 2009;30(19):3307-3317.

16. Okada M, Masuda M, Tanaka R, Miyatake K, Kuroda D, Furuzono T. Preparation of hydroxyapatite-nanocrystal-coated stainless steel, and its cell interaction. J Biomed Mater Res A. 2008;86(3):589-596.

17. Pezzatini S, Morbidelli L, Solito R, et al. Nanostructured HA crystals up-regulate FGF-2 expression and activity in microvascular endothelium promoting angiogenesis. Bone. 2007;41(4):523-534.

18. Zhao X, Ng S, Heng BC, et al. Cytotoxicity of hydroxyapatite nanoparticles is shape and cell dependent. Arch Toxicol. 2013;87(6): $1037-1052$.

19. Xie G, Sun J, Zhong G, Liu C, Wei J. Hydroxyapatite nanoparticles as a controlled-release carrier of BMP-2: absorption and release kinetics in vitro. J Mater Sci Mater Med. 2010;21(6):1875-1880.

20. Liu X, Sun J. Endothelial cells dysfunction induced by silica nanoparticles through oxidative stress via JNK/P53 and NF-kappaB pathways. Biomaterials. 2010;31(32):8198-8209.

21. Kang M, Lim CH, Han JH. Comparison of toxicity and deposition of nano-sized carbon black aerosol prepared with or without dispersing sonication. Toxicol Res. 2013;29(2):121-127.

22. Zhao X, Heng BC, Xiong S, et al. In vitro assessment of cellular responses to rod-shaped hydroxyapatite nanoparticles of varying lengths and surface areas. Nanotoxicology. 2011;5(2):182-194.

23. Xu Z, Liu C, Wei J, Sun J. Effects of four types of hydroxyapatite nanoparticles with different nanocrystal morphologies and sizes on apoptosis in rat osteoblasts. J Appl Toxicol. 2012;32(6):429-435.

24. Clift MJ, Gehr P, Rothen-Rutishauser B. Nanotoxicology: a perspective and discussion of whether or not in vitro testing is a valid alternative. Arch Toxicol. 2011;85(7):723-731.

25. Wan R, Mo Y, Zhang X, Chien S, Tollerud DJ, Zhang Q. Matrix metalloproteinase- 2 and -9 are induced differently by metal nanoparticles in human monocytes: The role of oxidative stress and protein tyrosine kinase activation. Toxicol Appl Pharmacol. 2008;233(2): 276-285. 
26. Oh WK, Kim S, Choi M, et al. Cellular uptake, cytotoxicity, and innate immune response of silica-titania hollow nanoparticles based on size and surface functionality. ACS Nano. 2010;4(9):5301-5313.

27. Kofler S, Nickel T, Weis M. Role of cytokines in cardiovascular diseases: a focus on endothelial responses to inflammation. Clin Sci (Lond). 2005;108(3):205-213.

28. Dessein PH, Joffe BI, Singh S. Biomarkers of endothelial dysfunction, cardiovascular risk factors and atherosclerosis in rheumatoid arthritis. Arthritis Res Ther. 2005;7(3):R634-R643.

29. Hou T, Tieu BC, Ray S, et al. Roles of IL-6-gp 130 signaling in vascular inflammation. Curr Cardiol Rev. 2008;4(3):179-192.

30. Pu FR, Williams RL, Markkula TK, Hunt JA. Expression of leukocyteendothelial cell adhesion molecules on monocyte adhesion to human endothelial cells on plasma treated PET and PTFE in vitro. Biomaterials 2002;23(24):4705-4718.

31. Rose SL, Babensee JE. Procoagulant phenotype of endothelial cells after coculture with biomaterial-treated blood cells. J Biomed Mater Res A. 2005;72(3):269-278.

32. Martinesi M, Bruni S, Stio M, Treves C, Bacci T, Borgioli F. Biocompatibility evaluation of surface-treated AISI 316L austenitic stainless steel in human cell cultures. J Biomed Mater Res A. 2007;80(1): $131-145$.

33. McDonald SM, Matheson LA, McBane JE, et al. Use of monocyte/ endothelial cell co-cultures (in vitro) and a subcutaneous implant mouse model (in vivo) to evaluate a degradable polar hydrophobic ionic polyurethane. J Cell Biochem. 2011;112(12):3762-3772.

34. Liu X, Xue Y, Sun J. Indirect induction of endothelial cell injury by PU- or PTFE-mediated activation of monocytes. J Biomater Sci Polym Ed. 2010;21(13):1783-1797.

35. Schanen BC, Karakoti AS, Seal S, Drake DR 3rd, Warren WL, Self WT. Exposure to titanium dioxide nanomaterials provokes inflammation of an in vitro human immune construct. ACS Nano. 2009;3(9):2523-2532.

36. Herseth J, Refsnes M, Låg M, Hetland G, Schwarze P. IL-1beta as a determinant in silica-induced cytokine responses in monocyteendothelial cell co-cultures. Hum Exp Toxicol. 2008;27(5):387-399.

37. Napierska D, Thomassen LC, Vanaudenaerde B, et al. Cytokine production by co-cultures exposed to monodisperse amorphous silica nanoparticles: the role of size and surface area. Toxicol Lett 2012;211(2):98-104.
38. Lawson C, Wolf S. ICAM-1 signaling in endothelial cells. Pharmacol Rep. 2009;61(1):22-32.

39. Muslin AJ. MAPK signalling in cardiovascular health and disease: molecular mechanisms and therapeutic targets. Clin Sci (Lond). 2008;115(7):203-218.

40. Mestas J, Ley K. Monocyte-endothelial cell interactions in the development of atherosclerosis. Trends Cardiovasc Med. 2008;18(6): 228-232.

41. Verma S, Anderson TJ. Fundamentals of endothelial function for the clinical cardiologist. Circulation. 2002;105(5):546-549.

42. Silverman MD, Zamora DO, Pan Y, et al. Cell adhesion molecule expression in cultured human iris endothelial cells. Invest Ophthalmol Vis Sci. 2001,42(12):2861-2866.

43. Makó V1, Czúcz J, Weiszhár Z, et al. Proinflammatory activation pattern of human umbilical vein endothelial cells induced by IL-1 $\beta$, TNF- $\alpha$, and LPS. Cytometry A. 2010;77(10):962-970.

44. Dong C, Davis RJ, Flavell RA. MAP kinases in the immune response. Annu Rev Immunol. 2002;20:55-72.

45. Zarubin T, Han J. Activation and signaling of the p38 MAP kinase pathway. Cell Res. 2005;15(1):11-18.

46. Mong PY, Petrulio C, Kaufman HL, Wang Q. Activation of Rho kinase by TNF-alpha is required for JNK activation in human pulmonary microvascular endothelial cells. J Immunol. 2008;180(1):550-558.

47. Richmond A. Nf-kappa B, chemokine gene transcription and tumour growth. Nat Rev Immunol. 2002;2(9):664-674.

48. Collins T, Read MA, Neish AS, Whitley MZ, Thanos D, Maniatis T. Transcriptional regulation of endothelial cell adhesion molecules: NF-kappa B and cytokine-inducible enhancers. FASEB J. 1995;9(10): 899-909.

49. Kuldo JM, Westra J, Asgeirsdóttir SA, et al. Differential effects of NF-\{kappa $\}$ B and p38 MAPK inhibitors and combinations thereof on TNF-\{alpha $\}$ - and IL-1\{beta $\}$-induced proinflammatory status of endothelial cells in vitro. Am J Physiol Cell Physiol. 2005;289(5): C1229-C1239.
International Journal of Nanomedicine

\section{Publish your work in this journal}

The International Journal of Nanomedicine is an international, peerreviewed journal focusing on the application of nanotechnology in diagnostics, therapeutics, and drug delivery systems throughout the biomedical field. This journal is indexed on PubMed Central,

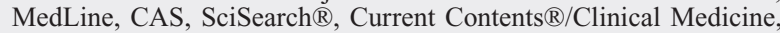

\section{Dovepress}

Journal Citation Reports/Science Edition, EMBase, Scopus and the Elsevier Bibliographic databases. The manuscript management system is completely online and includes a very quick and fair peer-review system, which is all easy to use. Visit http://www.dovepress.com/ testimonials.php to read real quotes from published authors. 